\title{
Melt behavior on the keyhole front during high speed laser welding.
}

\author{
I. Eriksson*, J. Powell, A.F.H. Kaplan \\ Lulea University of Technology - Sweden.
}

Luleå tekniska universitet

97187 Luleå

SWEDEN

ingemar.eriksson@Itu.se

tel: +46738139629

jpowell@laserexp.co.uk

alexander.kaplan@ltu.se

\begin{abstract}
The flow of molten metal on the front wall of a laser generated welding keyhole has been observed by high speed photography, optically measured by mapping the flow of ripples on the liquid surface and theoretically calculated. A clear downward flow can be observed and measured by a Particle Image Velocimetry algorithm. A theoretical calculation of the melt thickness on the keyhole front is also presented. Results indicate that the thickness of the liquid on the keyhole front is similar to that of the resolidified layer found in micrographs of the front if the laser is suddenly turned off. The measured surface ripple flow speeds are between two and four times as high as the theoretical average fluid flow rate.
\end{abstract}

\section{Keywords:}

Laser keyhole welding, PIV, Melt flow, High speed photography

*Corresponding author 
Authors version

Published in Optics and Lasers in Engineering

Volume 51, Issue 6, June 2013, Pages 735-740

http://dx.doi.org/10.1016/j.optlaseng.2013.01.008

\section{Introduction}

In laser keyhole welding the laser intensity is high enough to evaporate the metal and push a small capillary or 'keyhole' into the melt pool. Keyhole welds therefore have a high depth to width ratio. Laser keyhole welding can be divided into different regimes depending on the welding speed.[1] At low speeds the melt pool is rather large and the keyhole is surrounded by a considerable amount of melt on all sides. This gives the weld zone an approximately rotational symmetry.[2] As the welding speed increases there is less time for heat to conduct and thus there will be less melting in front of, and to the sides of, the keyhole. This results in a generally smaller melt volume and an asymmetrical keyhole/weldpool geometry with an inclined front.[3-5] The melt flowing down the front edge of the keyhole is involved in two basic mechanisms which drive the welding process;

a. The thin layer of melt on the front face of the keyhole passes heat forward in the direction of welding (by conduction) to melt the next layer of material.

b. The flow of hot liquid down the front face of the keyhole is the main mass transport process which creates the weld pool behind the keyhole - which solidifies to produce the weld.

As this paper will now demonstrate, it is possible to estimate the thickness of the melt from the thermal transport characteristics and, because we know the overall mass flow, this gives us an estimate of the average melt flow velocity. We can then compare this result with surface melt flow velocities measured from high speed videos.

The experiments presented in this paper are limited to bead on plate welds in $2.4 \mathrm{~mm}$ thick stainless steel 304 with a welding speed of $100 \mathrm{~mm} / \mathrm{s}$. As stainless steel has a rather low thermal conductivity (approximately 16-35 W/(m·K)) [6] this can be considered a high welding speed. At this speed for this material the thermal gradients will be very high and the melt thickness on the keyhole front will be thin. This paper considers the thermal transport within this thin melt and its associated fluid flow which eventually generates the weld.

The penetration depth of a keyhole laser weld is dependent on several welding parameters such as material type, welding speed, laser power, laser wavelength, laser spot size and 
Authors version

Published in Optics and Lasers in Engineering

Volume 51, Issue 6, June 2013, Pages 735-740

http://dx.doi.org/10.1016/j.optlaseng.2013.01.008

shielding gas. Often the trial and error method is used to reach the desired welding depth.

Empirical results show that when the welding speed is high enough to neglect the effects of thermal conduction in the base material, the welding depth $d$ is found to be proportional to $P / v$, where $P$ is the laser power and $v$ is the welding speed [7] (Assuming all other welding parameters are kept constant). A practical result of this is that you can reach the same welding depth at double the welding speed if you double the laser power - as long as you can avoid welding defects such as humping, undercut and spatter. As these defects are related to motions in the melt pool, it is important to understand the fluid flow during welding.

\section{An estimate of melt film thickness from thermal transport considerations.}

For stainless steel 304 the enthalpy increase from room temperature to melting (1700K) is $8,78 \mathrm{~J} \cdot \mathrm{mm}^{-3}$.[6] At a welding speed of $100 \mathrm{~mm} / \mathrm{s}$ the power requirement for melting the material is $878 \mathrm{~W}$ per $\mathrm{mm}^{2}$ of cross-section area. All this power must be transported from the front wall of the keyhole (where the laser beam is absorbed) to the melt-solid interface in front of the keyhole. The rate of thermal transport is dependant upon the thermal gradient between the front wall of the keyhole and the solid/liquid interface. As the maximum temperature of the melt surface is the boiling temperature (3080K at $1 \mathrm{~atm})$ the melt will have a thickness limited by the requirement to transfer the necessary power from the keyhole front to the melt-solid interface.

The ratio between convection and conduction (Péclet number) is unknown in this case, but if we look at pure conduction we can get a good estimation of the heat transfer charachteristics. The thermal conductivity of liquid steel is $17-22 \mathrm{~W} /(\mathrm{m} \cdot \mathrm{K})$ (depending on the temperature), giving a maximum thickness of melt of approximately $33 \mu \mathrm{m}$ if conduction was the only heat transfer process. Some convection will, of course, occur in the molten material, but a reasonable estimate of the maximum melt thickness for the required heat transfer would be $100 \mu \mathrm{m}$. A thicker layer would not be able to transport enough energy to melt the solid material in front of the keyhole. 
Authors version

Published in Optics and Lasers in Engineering

Volume 51, Issue 6, June 2013, Pages 735-740

http://dx.doi.org/10.1016/j.optlaseng.2013.01.008

\section{Fluid flow in the weld zone}

\subsection{Flow desciption}

To produce a weld it is not enough to simply melt the material. The molten material must pass from the front of the keyhole to the melt pool behind it and subsequently solidify into a weld. If a weld which has a cross section of $1 \mathrm{~mm}^{2}$ is welded at $100 \mathrm{~mm} / \mathrm{s}$, a total mass transfer of $100 \mathrm{~mm}^{3} / \mathrm{s}$ needs to be transported by a melt film with a thickness of $<100 \mu \mathrm{m}$, thus the liquid flow rate must be in the order of meters per second.

Previous empirical investigations of laser welding have shown that there is generally a downward flow on the front of the keyhole.[8-11] At high welding speed the surface of the liquid metal on the front wall of the keyhole is covered with small ripples or step like structures. With the help of a streak imaging technique developed by the authors [12] the velocity of these ripples has been measured and found to be in the order of $5-20 \mathrm{~m} / \mathrm{s}$.

The force driving the liquid metal from the front of the keyhole towards the melt pool behind the keyhole is the pressure created by evaporation on the keyhole wall [13]. When the irradiated surface is rippled the evaporation will not be uniform[14] but directed towards the light source and the vapor will affect the molten liquid film in a similar way to the jet used in laser cutting to propel melt out of the cut zone.[15] This principle is utilized in remote fusion cutting where the fluid flow away from the laser ejects material and produces a cut kerf without any assist gas [16]. The fluid flow of a thin liquid layer driven by a high velocity gas jet is a complicated two-phase problem that has been the subject of a considerable amount of research. [17-20] As the gas jet induces shear forces in the liquid, the waves produced exhibit strong non-linear behavior.[17] Fig 1 shows that the liquid/gas interaction goes through stages of stability (a) and wave growth (b) before non-linear waves (c) are established. The liquid flow is constant in the different zones (as we are considering an incompressible liquid).

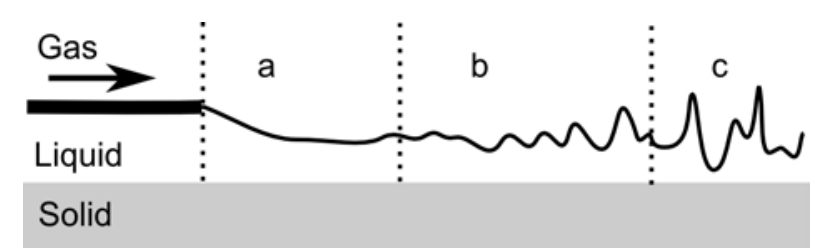

Figure 1. The development of ripples in a flowing liquid film.[17]a. Stabilization zone. $b$. Growing waves. c. Nonlinear waves 
Authors version

Published in Optics and Lasers in Engineering

Volume 51, Issue 6, June 2013, Pages 735-740

http://dx.doi.org/10.1016/j.optlaseng.2013.01.008

Investigations into the structure and shape of the nonlinear waves (Fig 1c) have shown that the wave crests move at the same speed as the main body of the wave.[19] This phenomenon has been experimentally confirmed for water flowing in a tube. Measuring the movement of the surface structure would therefore give a measurement of the fluid velocity in the fully developed ripples. However, on the laser welding keyhole front the liquid metal has a temperature range from the melting temperature $(1700 \mathrm{~K})$ up to the evaporation temperature (3000K). Over this temperature range the viscosity of the stainless steel changes from 6.4mPa $\cdot \mathrm{s}$ to $1.6 \mathrm{mPa} \cdot \mathrm{s}$.[6] This makes it hard to justify the simplifications used for the analysis of water/gas interactions, but an estimate of flow directions and velocities is possible by measuring the movement of the molten surface.

\subsection{Flow velocity measurements}

A Photron SA1 high speed camera was mounted at a 45 degree inclination angle (see Fig 4a), and a video of the keyhole front was captured at 180000 frames per second. To reduce the motion blur the exposure time was set to $1 \mu \mathrm{s}$. The camera imaged a $4.2 \mathrm{x} 3 \mathrm{~mm}$ area from a distance of $400 \mathrm{~mm}$, giving an almost orthographic image. To increase the frame rate the image resolution was decreased to $128 \times 128$ pixels, giving a spatial resolution of $23 \mu \mathrm{m}$ per pixel. With this set-up a movement of 1 pixel between two frames would be equivalent to $4.2 \mathrm{~m} / \mathrm{s}$ flow velocity. Objects and artifacts moving faster than $20 \mathrm{~m} / \mathrm{s}$ would experience some motion blur smearing out the details. As the back wall of the keyhole obscured the camera view, only a limited depth down the keyhole front could be observed. In Fig 4 this depth is shown as $\mathrm{X}$.

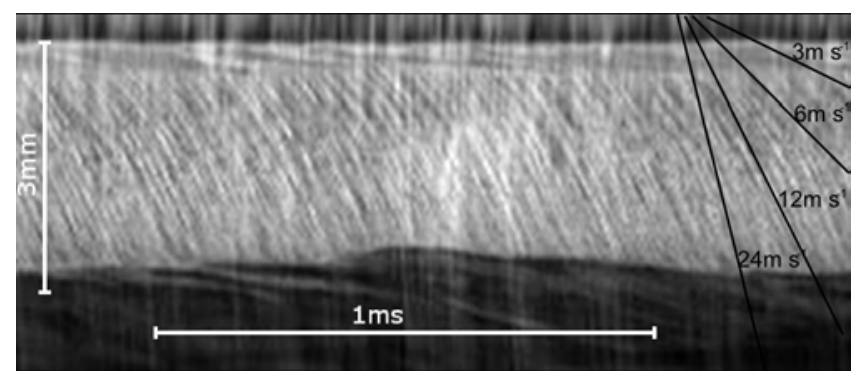

Figure 2. Streak image [21]

Using the streak image technique previously reported the most dominant downward velocity in each column of the image could be measured.[21] A column from each frame is extracted and stacked as a streak image as in Fig 2. In this streak image a moving object will produce a inclined line, and the vertical velocity component of the object can be measured by measuring 
the inclination angle of the line in the streak image. By using a 2D-FFT transform the most common velocity in the streak image can be identified.

In Fig 3 the most dominant velocity in a 4000frame long video is displayed for different columns in the video sequence. In this particular experiment the welding speed was $100 \mathrm{~mm} / \mathrm{s}$, and the laser power $6 \mathrm{~kW}$. The $4-\sigma$ diameter of the laser beam was measured as $0,9 \mathrm{~mm}$ on the top surface of the plate. The keyhole extends from columns 70 to 95 in Fig 3, outside this range the apparent peaks are due to image noise. The dominant downward velocity has an almost constant value of $7.5 \mathrm{~m} / \mathrm{s}$ over the entire width of the keyhole front surface.



Figure 3. Downward flow in different columns down the weld front

For high welding speeds the measured downward velocity was found to have a linear dependence on laser power, and to be virtually independent of welding speed.[21] This indicates that the downward flow is driven by the local evaporation pressure on the keyhole front. This evaporation pressure is closely related to the surface temperature and the irradiance of the laser beam.[22] 

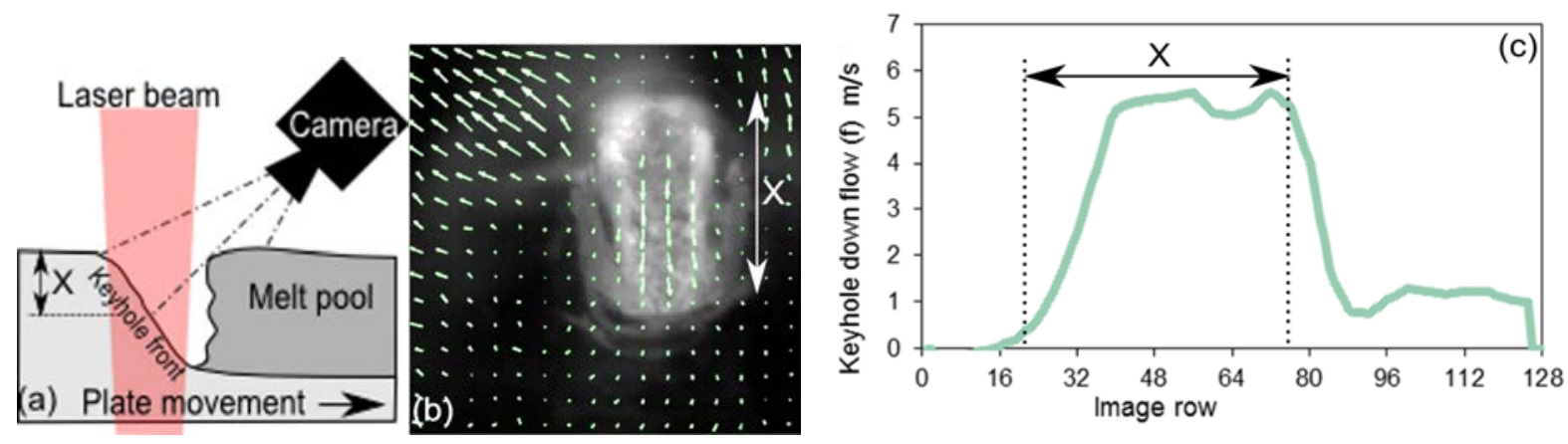

Figure 4. a: The camera's view of the weld zone. b:Frame with average PIV calculation of 200 frames. c: flow velocity down the centre line of the keyhole front measured from PIV.

To examine the characteristics of the fluid flow further, a Particle Image Velocimetry (PIV) toolbox in MATLAB (called PIVlab) was utilized. The PIV algorithm computes the cross correlation of 8x8 pixel segments in two consecutive frames using the FFT method.[23] After the cross correlation calculation the local translation between the images is found by a 2DGauss interpolation scheme. The PIV calculations result in a vector field indicating the magnitude and direction of the local movement in the image, although in areas with low image texture the image noise can have a large impact. Fig 4b shows a frame of the high speed video with an overlay of an average of 200 PIV calculations. This demonstrates that the flow on the keyhole front is almost entirely downward. The PIV results can be used to create the type of surface velocity information presented in Fig 4c. In this figure we can see the velocity results for the centre line of the flow. Image rows 20 - 75 show that the flow initially accellerates from the top of the keyhole and then reaches a steady downwards velocity of approximately 5.5m/s. (Image rows above 75 are measurements of the flow on top of the melt pool which occludes the cameras view of the keyhole front - see fig 4a). This value is lower than the velocity measuered by the streak image method because the PIV calculations show zero velocity in areas with low contrast. The measured velocity in Fig 3 and Fig 4 is calibrated to a vertical keyhole front. In the case of an inclined keyhole the actual velocity could be up to $29,3 \%$ lower than measured. 
Authors version

Published in Optics and Lasers in Engineering

Volume 51, Issue 6, June 2013, Pages 735-740

http://dx.doi.org/10.1016/j.optlaseng.2013.01.008

\section{Keyhole front inclination and melt film thickness}

During laser welding at high speed the inclination angle of the keyhole front is clearly related to the welding parameters. $[5,24]$ An increase in welding speed or a decrease in laser power will increase the inclination angle. Changing the beam spot size will change both the laser irradiance and the interaction time with the material. This combination changes the inclination angle and the penetration depth. [25] An estimate of the inclination angle of the keyhole front can be found by taking a longitudinal section of an interrupted weld (ie a weld where the laser is suddenly turned off - see Fig 5a). When the laser beam is switched off the shape of the keyhole front is "frozen".
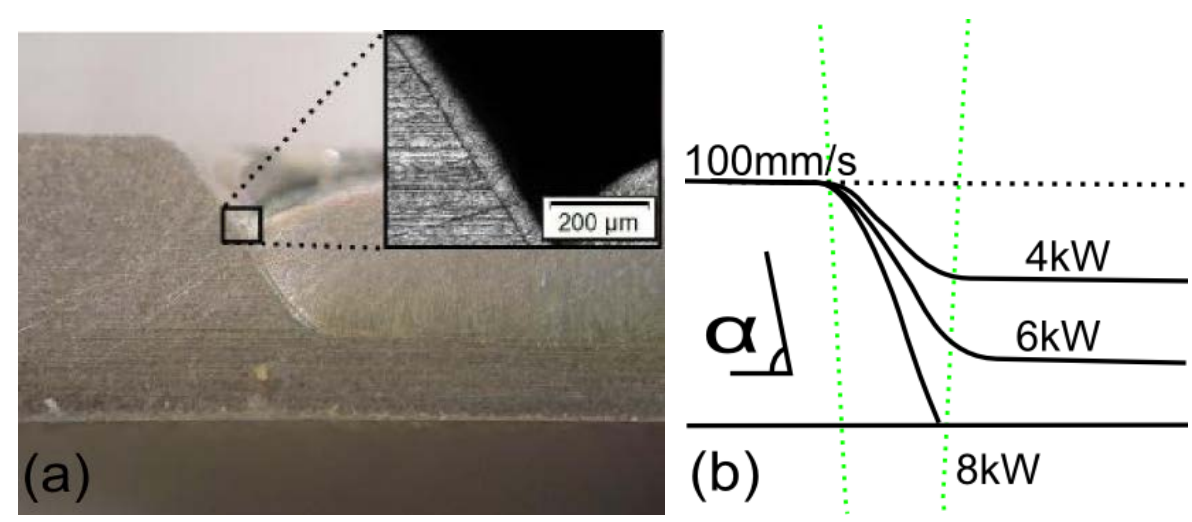

Figure 5 a: Longitudinal cut of weld. $b$ : Inclination ( $\alpha$ ) for different laser powers

Fig 5 demonstrates that, for a given laser-material combination the inclination angle of the keyhole front is determined by the width of the laser beam and the penetration depth of the weld zone. - so the keyhole front becomes more vertical as laser power is increased or welding speed is reduced. In our calculations about flow velocity it is important to bear in mind that the view angle of the high speed camera changes if the keyhole front inclination changes.

The close up micrograph of the keyhole front in Fig 5a shows a thin section resolidified material approximately $50 \mu \mathrm{m}$ thick. To find out if this is a true representation of the melt thickness during welding, further analysis was carried out.

Using the finite difference method described by Incropera and DeWitt [26] the cooling curve of the free surface of a 50 $\mu \mathrm{m}$ thick liquid layer was calculated. The 1-D model with 200 elements and a step length of $10 \mu \mathrm{m}$ was solved explicit with a time step length of $0.05 \mathrm{~ns}$. The 
thermal diffusivity [6] was divided by 4 in the interval 1700-1750K to take account of the latent heat of fusion. The initial temperature in the solid part of the model was the steady state temperature gradient formed during welding. The initial temperature in the liquid layer was set to a linear $2500-1700 \mathrm{~K}$ gradient. The result of this calculation- presented in Fig 6a shows that the freezing time of the thin melt in the absence of the laser beam would be of the order of $0.5 \mathrm{~ms}$. Some flow could, of course, occur during this time but it would be hampered by two phenomena. The first of these is that as soon as the laser beam is extinguished the directional evaporation on the laser irradiated side of any ripples would cease - removing the thrust behind downward flow. The other point is that, during solidification the average viscosity[6] of the molten layer would rise rapidly (see Fig 6b) which would further impede flow.

From these considerations it can be estimated that the thickness of the melt frozen on the keyhole surface (see fig 5a) is a reasonable approximation of the melt thickness which exists during welding.
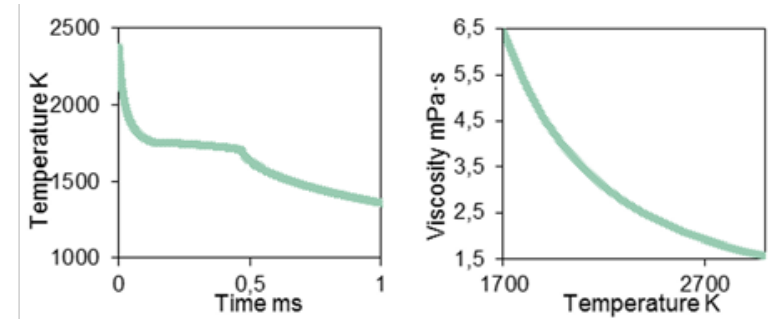

Figure 6. a: Modelled temperature at the surface during cooling. b: Viscosity of stainless steel 304 as a function of temperature[6]. 


\section{Theoretical Model}

High speed camera observations of the keyhole front show that the dominant flow is down the front face of the keyhole.[12] Toward the sides of the keyhole, lower flow speeds and horizontal shear forces will introduce some horizontal fluid flow, but in the thin strip in the center of the keyhole front the fluid flow can be assumed to be flowing only downward. In Fig 7 the width of this strip is denoted $w$.

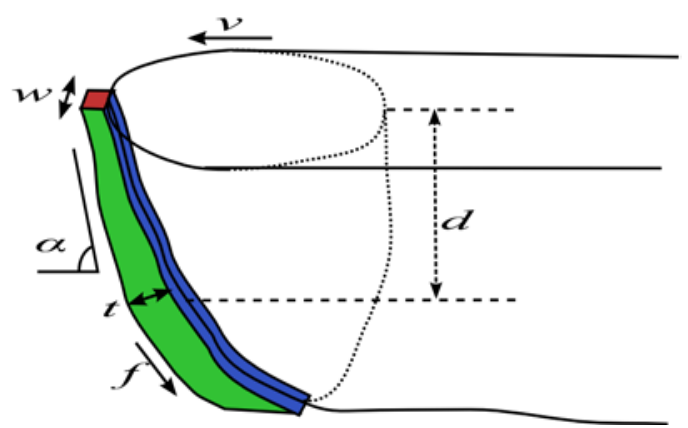

Figure7. Model for fluid flow on keyhole front wall

The volume $V$ flowing past a point at a depth (into the keyhole) $d$ is the sum of all the flow from depth 0 to depth $d$. This is equal to a flow of width $w$ and depth d multiplied by the welding speed. As all material in this strip is flowing downward the entire molten volume must flow past a position at a depth $d$.

$$
V_{d}=v \cdot w \cdot d
$$

As an example; at a welding speed of $100 \mathrm{~mm} / \mathrm{s}$ and a width of $0.1 \mathrm{~mm}$ the volumetric fluid flow rate at $1 \mathrm{~mm}$ depth is $10 \mathrm{~mm}^{3} / \mathrm{s}$.

The volume flowing past a position on the keyhole front can also be calculated from the film thickness $t$ at that depth, multiplied by the width and the average film flow velocity $f_{d}$.

$$
V_{d}=w \cdot t_{d} \cdot f_{d}
$$

Equation (1) and (2) can be combined to give the downward fluid flow velocity at a given depth. According to equation (3) a film thickness of $50 \mu \mathrm{m}$ at $1 \mathrm{~mm}$ depth with a weldng speed of $100 \mathrm{~mm} / \mathrm{s}$ would require an average fluid velocity of $2 \mathrm{~m} / \mathrm{s}$. 


$$
f_{d}=\frac{v \cdot d}{t_{d}}
$$

The measurements attained by high speed camera only observe structures on the surface of the fluid film, and the flow on the surface will undoubtedly be higher than the average flow. In equation (4) a constant $k$ has been introduced to calculate the flow velocity on the surface of the fluid film. In gas driven nonlinear waves of water in tubes the value of $k$ has been shown to be close to 1.[19] In the case of a purely laminar flow with the driving force acting on the top surface (Couette flow) the value of $k$ would be 2. Both these values are based on a constant viscosity but on the keyhole front there is a viscosity gradient, thus the flow will be more complicated and a higher value of $k$ expected.

$$
f_{\text {surface }}=k \cdot \frac{v \cdot d}{t_{d}}
$$


Authors version

Published in Optics and Lasers in Engineering

Volume 51, Issue 6, June 2013, Pages 735-740

http://dx.doi.org/10.1016/j.optlaseng.2013.01.008

\section{Discussion}

In Fig 8 the inclination angle of the keyhole front and the thickness of the resolidified layer seen in Fig 5a are plotted for different positions down the keyhole front surface. This is the resulting inclination angle from welding at $100 \mathrm{~mm} / \mathrm{s}$ with a laser power of $6 \mathrm{kWand}$ a beam diameter of $0.9 \mathrm{~mm}$.

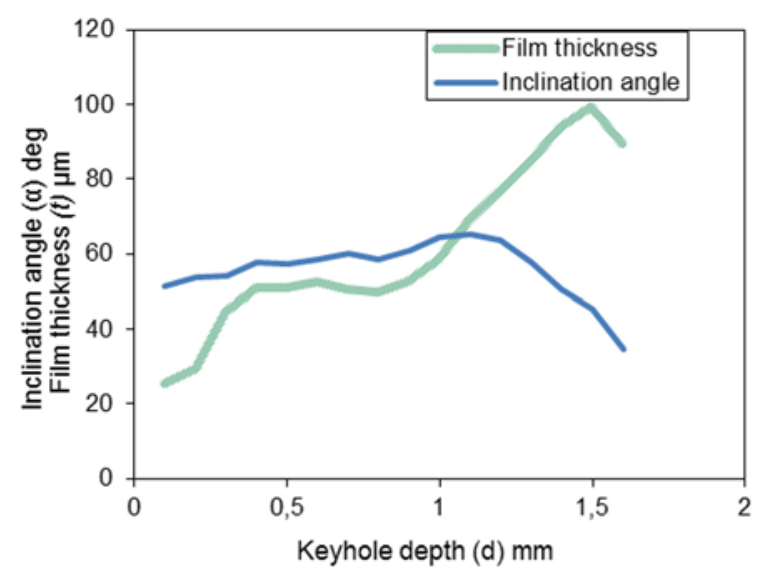

Figure 8. Measured layer thickness and inclination angle along the keyhole front in Fig5a.

Using these measured film thicknesses in equation (4) the flow velocity on the keyhole front can be calculated and compared to the measured velocities in Fig 4c. In Fig 9 the measured PIV velocity has been corrected to the inclination angle in Fig 8 and is thus lower than in Fig 4c where the keyhole front was assumed to be at 90 degree inclination. As the inclination angle changes the projection angle towards the camera, and observed depth has to be adjusted to the keyhole shape in Fig 5. As seen in Fig 4a the camera can only observe the front to a depth of $\mathrm{X}$, in this case approximately $1,1 \mathrm{~mm}$, therefor only PIV values to this depth are shown in Fig9. The measured value shows the surface velocity $f_{\text {surface, }}$, therefore we need to find the ratio $k$ between surface velocities and average velocities.

It was mentioned in section 3.1 that the assumption that ripple velocity is equal to bulk flow velocity, whilst being valid for thin films of water would not apply to the melt flowing down the front face of a keyhole where there is a temperature gradient from the melting point (at the melt-solid boundary) to the boiling point (on the outer melt face). This point is clearly demonstrated in Fig 9 where it can be seen that the measured surface ripple velocity is up to four times as fast as the calculated average flow rate. 


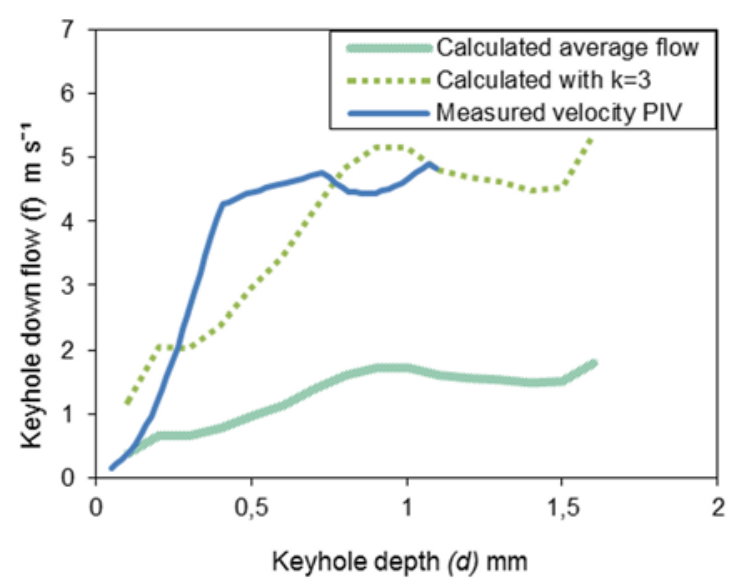

Figure 9. Flow velocities on the keyhole front at different depths into the keyhole.

This result follows the trend we would expect from a fluid with a high thermal, and therefore high viscosity, gradient (see Fig 6b). From these initial results it appears that the surface ripple velocity rapidly accelerates to a high value ( $4.5 \mathrm{~m} / \mathrm{s}$ in this case) and rises only gradually thereafter. The average velocity of the flow rises steadily and, after the first millimeter or so, reaches a velocity which is approximately $33 \%(\mathrm{k}=3)$ of the surface ripple speed (within the constraints of the experimental view of the keyhole front - see fig 4a). This result is valuable in that it shows that the monitoring of surface ripple speeds can give a considerable amount of information about the underlying flow of the melt. 


\section{Conclusions}

In our example (welding SS304 at 100mm/s) the thickness of the molten material on the keyhole front is limited by thermo-dynamical properties to approximately $100 \mu \mathrm{m}$. This implies that the fluid flows on the front must be of the order of meters per second. A micrograph of the keyhole front revealed a resolidified layer of similar thickness - in our case from approximately $20 \mu \mathrm{m}$ close to the top of the keyhole to approximately $100 \mu \mathrm{m}$ further down.

High speed imaging combined with Particle Image Velocimetry can be used to produce a velocity map of the flow of surface ripples inside laser welding keyholes. The downward flow speed of ripples on the liquid surface were measured in our case as rising from close to zero near the top of the keyhole to approximately $4.5 \mathrm{~m} / \mathrm{s}$ after a keyhole depth of $0.4 \mathrm{~mm}$. The flow is continuously downward across the keyhole.

Surface ripple flow speeds are between two and four times as high as the theoretical average fluid flow rate. This can be explained by a velocity gradient in the fluid film due to a strong viscosity gradient.

\section{Acknowledgement}

This research has been carried out in the FiberTubeAdvanced project, funded by VINNOVA (The Swedish Agency for Innovation Systems) and Jernkontoret (The Swedish Steel Producers Association), project no. 34013. 


\section{References}

[1] Fabbro R. Melt pool and keyhole behaviour analysis for deep penetration laser welding. $J$ Phys D 2010;43:445501.

[2] Dowden J, Postacioglu N, Davis M, Kapadia P. A keyhole model in penetration welding with a laser. $J$ Phys $D$ 1987;20:36.

[3] Matsunawa A, Semak V. The simulation of front keyhole wall dynamics during laser welding. J Phys D 1997;30:798.

[4] Fabbro R, Chouf K. Dynamical description of the keyhole in deep penetration laser welding. J Laser Appl 2000;12:142.

[5] Fabbro R, Slimani S, Coste F, Briand F. Study of keyhole behaviour for full penetration Nd-Yag CW laser welding. J Phys D 2005;38:1881.

[6] Leibowitz L, Chang E, Chasanov M, Gibby R, Kim C, Millunzi A, et al. Properties for LMFBR safety analysis. Argonne National Labs 1976.

[7] Locke E, Hoag E, Hella R. Deep penetration welding with high-power CO2lasers. IEEE Journal of Quantum Electronics 1972;8(2):132-5.

[8] Arata Y, Abe N, Oda T. Fundamental phenomena in high power CO 2 laser welding (Report I). Trans JWRI 1985;14(1):5-11.

[9] Matsunawa A. Problems and solutions in deep penetration laser welding. Science and Technology of Welding and Joining 2001;6(6):351-4.

[10] Semak V, Hopkins J, McCay M, McCay T. Melt pool dynamics during laser welding. $J$ Phys D 1995;28:2443.

[11] Hirano K, Fabbro R, Muller M. Experimental determination of temperature threshold for melt surface deformation during laser interaction on iron at atmospheric pressure. $J$ Phys $D$ 2011;44:435402.

[12] Eriksson I, Gren P, Powell J, Kaplan AFH. New high-speed photography technique for observation of fluid flow in laser welding. Optical Engineering 2010;49:100503.

[13] Semak V, Matsunawa A. The role of recoil pressure in energy balance during laser materials processing. $J$ Phys D 1997;30:2541.

[14] Kaplan AFH. Local absorptivity modulation of a $1 \mu$ m-laser beam through surface waviness. Appl Surf Sci 2012.

[15] Vicanek M, Simon G, Urbassek H, Decker I. Hydrodynamical instability of melt flow in laser cutting. J Phys D 1987;20:140. 
[16] Schober A, Musiol J, Daub R, Feil J, Zaeh M. Experimental Investigation of the Cutting Front Angle during Remote Fusion Cutting. Physics Procedia 2012;39:204-12.

[17] Alekseenko S, Aktershev S, Cherdantsev A, Kharlamov S, Markovich D. Primary instabilities of liquid film flow sheared by turbulent gas stream. Int J Multiphase Flow 2009;35(7):617-27.

[18] Asali J, Hanratty T. Ripples generated on a liquid film at high gas velocities. Int $J$ Multiphase Flow 1993;19(2):229-43.

[19] Hewitt G, Jayanti S, Hope C. Structure of thin liquid films in gas-liquid horizontal flow. Int J Multiphase Flow 1990;16(6):951-7.

[20] Webb D, Hewitt G. Downwards co-current annular flow. Int J Multiphase Flow 1975;2(1):35-49.

[21] Eriksson I, Powell J, Kaplan AFH. Measurements of fluid flow on keyhole front during laser welding. Science and Technology of Welding and Joining 2011;16(7):636-41.

[22] Semak VV, Knorovsky G, MacCallum D. On the possibility of microwelding with laser beams. J Phys D 2003;36(17):2170.

[23] Willert C, Gharib M. Digital particle image velocimetry. Exp Fluids 1991;10(4):181-93.

[24] Pan Y, Richardson I. Keyhole behaviour during laser welding of zinc-coated steel. J Phys D 2011;44:045502.

[25] Suder W, Williams S. Investigation of the effects of basic laser material interaction parameters in laser welding. J Laser Appl 2012;24:032009.

[26] Incropera F, DeWitt D. Introduction to heat transfer. Introduction to heat transfer. 4th ed. New York: John Wiley and Sons Inc.; 2001. p. 281. 